\title{
Spectroscopic Binaries in the Open Cluster M67
}

\author{
DAVID W. LATHAM \\ Harvard-Smithsonian Center for Astrophysics, 60 Garden Street, \\ Cambridge, MA 02138, USA \\ ROBERT D. MATHIEU
}

Department of Astronomy, University of Wisconsin, 475 Charter Street, Madison, WI 53706, USA

\author{
ALEJANDRA A. E. MILONE \\ Harvard-Smithsonian Center for Astrophysics, 60 Garden Street, \\ Cambridge, MA 02138, USA, and \\ Cördoba Observatory, National University of Cördoba, Laprida 854, 5000 \\ Cördoba, Argentina
}

\section{ROBERT J. DAVIS}

Harvard-Smithsonian Center for Astrophysics, 60 Garden Street, Cambridge, MA 02138, USA

\begin{abstract}
For almost 400 members of M67 we have accumulated about 5,000 precise radial velocities. Already we have orbital solutions for more than 32 spectroscopic binaries in M67. Many of these orbits were derived by combining the Palomar and CfA observations, thus extending the time coverage to more than 20 years. The distribution of eccentricity versus period shows evidence for tidal circularization on the main sequence. The transition from circular orbits is fairly clean. Excluding the blue stragglers, the first eccentric orbit has a period of 11.0 days, while the last circular orbit has a period of 12.4 days. For longer periods the distribution of eccentricity is the same as for field stars. The blue straggler S1284 has an eccentric orbit despite its short period of 4.2 days.
\end{abstract}

In 1971 Roger Griffin and Jim Gunn began monitoring the radial velocities of most of the members brighter than the main-sequence turnoff in the old open cluster M67, primarily using the 200-inch Hale Telescope. In 1982, just as the sequence of observations at Palomar was ending, Dave Latham and Bob Mathieu began monitoring many of the same stars with the $1.5-\mathrm{m}$ Tillinghast Reflector and the Multiple-Mirror Telescope on Mount Hopkins. The Palomar and Mount Hopkins data sets were successfully merged, together with some additional CORAVEL velocities kindly provided by Michel Mayor, to obtain 20 years of time coverage (e.g., see Mathieu et al. 1986). Based on these data, orbital solutions have been published (Mathieu et al. 1990) for 22 spectroscopic binaries. The $\mathrm{M} 67$ survey is now being extended to $\mathrm{V}=15.5$ with the telescopes at Mount Hopkins. Already we have $\mathbf{1 3}$ additional orbital solutions, with the promise of many more to come.

In Table 1 we list the period and eccentricity for 34 spectroscopic binaries which are members of M67. For the binaries with circular orbits, Mathieu et al. (1990) adopted an eccentricity of 0.0 . Here we have allowed the eccentricity to be a free parameter in the orbital solution, so that the estimated error in the eccentricity may be used to judge whether the eccentricity is significantly 
different from circular.

In Figure 1 we plot eccentricity versus log period for the M67 binaries that have orbital solutions. All the binaries with periods shorter than 11 days have circular orbits, presumably due to tidal circularization (e.g., see Mathieu et al. 1992). The transition from circular to eccentric orbits is fairly clean. The last circular orbit has a period of 12.45 days, somewhat longer than the period of 11.02 days for the first eccentric orbit.

TABLE 1. M67 binaries

\begin{tabular}{|c|c|c|c|c|c|}
\hline Name & $V$ & $B-V$ & Period & Eccentricity & Reference \\
\hline S 1250 & 9.71 & 1.35 & 4410.000 & $0.50 \pm 0.03$ & MLG90 \\
\hline S 1221 & 10.76 & 1.13 & 6445.000 & $0.031 \pm 0.027$ & CfA \\
\hline S 1237 & 10.78 & 0.94 & 697.800 & $0.105 \pm 0.015$ & MLG90 \\
\hline S 1284 & 11.04 & 0.22 & 4.1828 & $0.266 \pm 0.045$ & M91 \\
\hline S 1072 & 11.32 & 0.63 & 1495.000 & $0.32 \pm 0.07$ & MLG90 \\
\hline S 1040 & 11.52 & 0.88 & 42.8271 & $0.027 \pm 0.028$ & CfA \\
\hline S 1264 & 11.74 & 0.92 & 353.900 & $0.383 \pm 0.013$ & MLG90 \\
\hline S 1053 & 12.19 & 0.84 & 123.39 & $0.486 \pm 0.018$ & MLG90 \\
\hline S 2206 & 12.30 & 0.84 & 18.377 & $0.341 \pm 0.065$ & MLG90 \\
\hline S 1272 & 12.52 & 0.65 & 11.0215 & $0.262 \pm 0.007$ & MLG90 \\
\hline S 1045 & 12.55 & 0.59 & 7.64521 & $0.007 \pm 0.005$ & CfA \\
\hline S 251 & 12.57 & 0.67 & 948.000 & $0.554 \pm 0.024$ & MLG90 \\
\hline S 1285 & 12.59 & 0.65 & 277.800 & $0.19 \pm 0.03$ & MLG90 \\
\hline S 999 & 12.60 & 0.78 & 10.05525 & $0.014 \pm 0.020$ & CfA \\
\hline S 1234 & 12.66 & 0.59 & 4.35563 & $0.058 \pm 0.023$ & MLG90 \\
\hline S 1000 & 12.69 & 0.88 & 531.1 & $0.092 \pm 0.018$ & MLG90 \\
\hline S 1242 & 12.70 & 0.72 & 31.7797 & $0.664 \pm 0.018$ & MLG90 \\
\hline S 1024 & 12.72 & 0.57 & 7.15961 & $0.005 \pm 0.005$ & CfA \\
\hline S 1508 & 12.73 & 0.70 & 25.866 & $0.441 \pm 0.007$ & MLG90 \\
\hline S 986 & 12.74 & 0.56 & 10.3386 & $0.006 \pm 0.008$ & CfA \\
\hline S 1216 & 12.75 & 0.56 & 60.445 & $0.451 \pm 0.007$ & MLG90 \\
\hline S 821 & 12.85 & 0.55 & 584.16 & $0.793 \pm 0.012$ & CfA \\
\hline S 1292 & 13.24 & 0.62 & 100.41 & $0.034 \pm 0.028$ & CfA \\
\hline S 990 & 13.45 & 0.55 & 41.847 & $0.352 \pm 0.018$ & CfA \\
\hline S 973 & 13.49 & 0.56 & 40.420 & $0.199 \pm 0.020$ & CffA \\
\hline S 1063 & 13.52 & 1.07 & 18.3874 & $0.217 \pm 0.014$ & CfA \\
\hline S 1009 & 13.70 & 0.56 & 5.9531 & $0.014 \pm 0.015$ & CfA \\
\hline S 1224W & 13.7 & & 12.448 & $0.059 \pm 0.030$ & CfA \\
\hline S 1070 & 13.98 & 0.61 & 2.66061 & $0.010 \pm 0.015$ & $\mathrm{CfA}$ \\
\hline S 1247 & 14.05 & 0.61 & 69.77 & $0.056 \pm 0.010$ & CfA \\
\hline S 1014 & 14.11 & 0.81 & 16.219 & $0.307 \pm 0.011$ & CfA \\
\hline S 981 & 14.14 & 0.62 & 55.831 & $0.508 \pm 0.047$ & CfA \\
\hline S 2222 & 14.76 & 0.82 & 25.6712 & $0.544 \pm 0.011$ & CfA \\
\hline S 810 & 15.16 & 0.92 & 10.151947 & $0.014 \pm 0.021$ & CfA \\
\hline
\end{tabular}




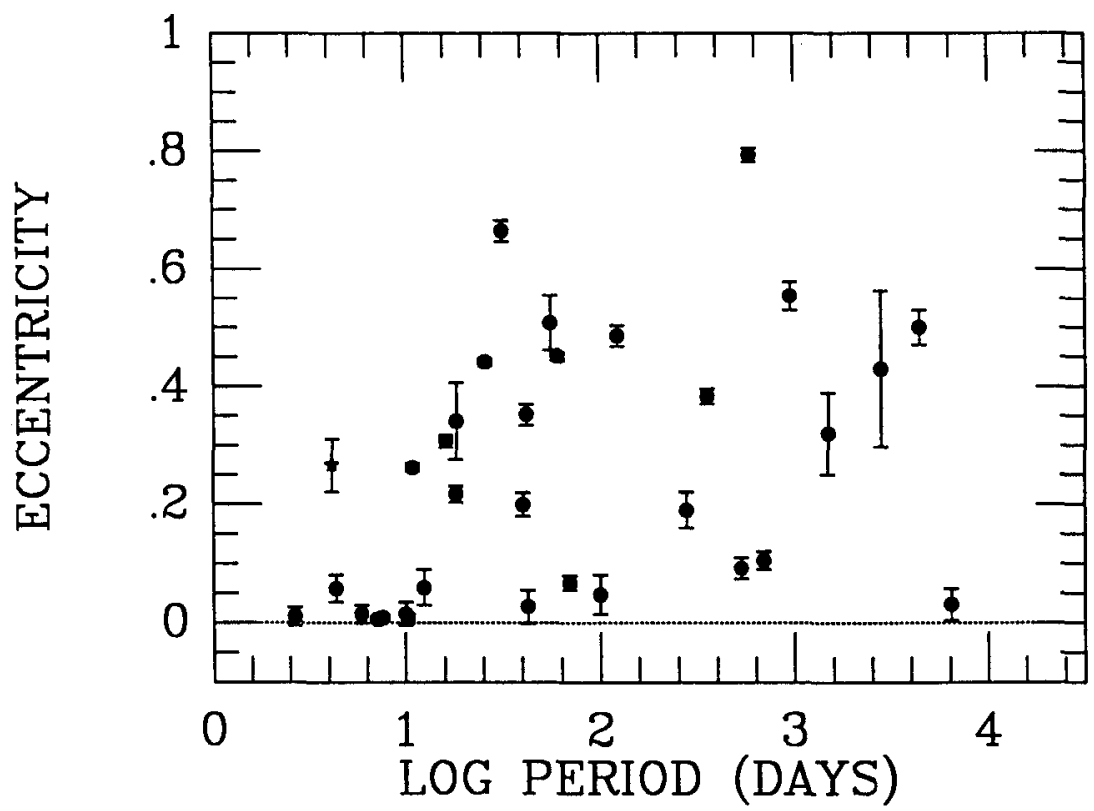

FIGURE 1. Eccentricity versus $\log$ period for 34 binaries in M67.

The 23 M67 binaries with periods longer than 12.4 days show a wide spread of eccentricities, with a mean eccentricity of $0.33 \pm 0.05$. This is somewhat smaller than the mean eccentricity of $0.42 \pm 0.03$ for a sample of 44 binaries with period longer than 14 days found among 164 nearby solar-type field dwarfs (Duquennoy \& Mayor 1991). Two of the main-sequence binaries in M67 with fairly long periods have nearly circular orbits: $\$ 1292$ with period 100 days and S1247 with period 70 days, while two of the evolved binaries with fairly long periods have nearly circular orbits: S1221 with period 6445 days, and S1040 with period 43 days. Perhaps these binaries were formed with nearly circular orbits, or perhaps tidal circularization has been more effective due to a more extensive convective zone if the star or its companion has evolved off the main sequence.

\section{REFERENCES}

Duquennoy, A., \& Mayor, M. 1991, $A \mathscr{E A}$ A, 248, 485

Mathieu, R.D., Duquennoy, A., Latham, D.W., Mayor, M., Mazeh, T., Mermilliod, J.C. 1992, in Workshop on Binaries as Tracers of Stellar Formation and Evolution, eds. A. Duquennoy and M. Mayor (Cambridge, Cambridge University Press), in press

Mathieu, R.D., Latham, D.W. \& Griffin, R.F. 1990, $A J, 100,1859$

Mathieu. R.D., Latham, D.W., Griffin, R.F., \& Gunn, J.E. 1986, $A J$, 92, 1100 